\title{
Piecewise Implicit Differential Systems
}

\author{
Bruno D. Lopes ${ }^{1}$ • Paulo R. da Silva ${ }^{1}$. \\ Marco A. Teixeira ${ }^{2,3}$
}

Received: 13 November 2015 / Revised: 26 April 2016 / Published online: 31 May 2016

C) Springer Science+Business Media New York 2016

Abstract In this article we deal with non-smooth dynamical systems expressed by a piecewise first order implicit differential equations of the form

$$
\dot{x}=1, \quad(\dot{y})^{2}=\left\{\begin{array}{lll}
g_{1}(x, y) & \text { if } \varphi(x, y) \geq 0 \\
g_{2}(x, y) & \text { if } \varphi(x, y) \leq 0
\end{array},\right.
$$

where $g_{1}, g_{2}, \varphi: U \rightarrow \mathbb{R}$ are smooth functions and $U \subseteq \mathbb{R}^{2}$ is an open set. The main concern is to study sliding modes of such systems around some typical singularities. The novelty of our approach is that some singular perturbation problems of the form

$$
\dot{x}=f(x, y, \varepsilon), \quad(\varepsilon \dot{y})^{2}=g(x, y, \varepsilon)
$$

arise when the Sotomayor-Teixeira regularization is applied with $(x, y) \in U, \varepsilon \geq 0$, and $f, g$ smooth in all variables.

Keywords Non-smooth dynamical system · Implicit differential equation · Singular perturbation $\cdot$ Sliding vector fields

\section{Introduction}

\subsection{Motivation and Historical Facts}

Since our early paper [13] considerable efforts have been made to develop general-purpose tools for the study of qualitative geometric analysis of non-smooth systems. Some of theo-

Bruno D. Lopes

brunodomicianolopes@gmail.com

1 IBILCE-UNESP, Rua C. Colombo, 2265, CEP 15054-000 S. J. Rio Preto, São Paulo, Brazil

2 IMECC-UNICAMP, CEP 13081-970 Campinas, São Paulo, Brazil

3 UFSCAR, CEP 18052-780 Sorocaba, São Paulo, Brazil 
retical tools one may employ include: singularity theory, regularization process, blowing-up method and singular perturbation theory.

In recent years we have detected a significant development of the research related to piecewise smooth dynamical systems. These systems sound interesting for two reasons: first they behave in ways outside of smooth systems, and second they are found in many important application areas, for example, physics and control theory, among others. The book [9] and the papers $[2-4,12]$ give an excellent introduction to the subject.

A consistent comparison between smooth, continuous non-smooth and discontinuous non-smooth systems could be a leading theme of research not only by the beauty of mathematical results, but also because of the huge applicability of the results in practical problems. We are interested in the particular subject: non-smooth dynamical systems expressed by a piecewise first order implicit differential equations. As far as we know, these systems have not been considered in the literature, so far.

Let $F$ be a $C^{2}$ map defined around $q_{0}=\left(x_{0}, y_{0}, \dot{y_{0}}\right) \in \mathbb{R}^{3}$. It is well known that an implicity equation

$$
F(x, y, \dot{y})=0,
$$

can be solved as $\dot{y}=f(x, y)$ provided that $D_{\dot{y}} F\left(q_{0}\right) \neq 0$. In this article solutions of the equations are investigated without such condition. More precisely we consider equations satisfying $D_{\dot{y}} F\left(q_{0}\right)=0$ but assuming that $D_{\dot{y} \dot{y}} F\left(q_{0}\right) \neq 0$. In this sense, local coordinates can be considered such that

$$
F(x, y, \dot{y})=(\dot{y})^{2}-g(x, y) .
$$

Many authors have contributed to implicit equations of first order and related problems, e.g. $[1,5,7,8]$. We also refer $[18]$ as an inspiring reading on the subject.

\subsection{Goal}

We consider piecewise implicit equations having the form

$$
(\dot{y})^{2}= \begin{cases}g_{1}(x, y) & \text { if } \quad \varphi(x, y) \geq 0 \\ g_{2}(x, y) & \text { if } \varphi(x, y) \leq 0,\end{cases}
$$

where $g_{i}: U \rightarrow \mathbb{R}, i=1,2$, and $\varphi: U \rightarrow \mathbb{R}$ are smooth functions, and $U \subseteq \mathbb{R}^{2}$ is an open set (see Figs. 1, 2). Our concern is to study the dynamics of such systems around the curve $\varphi(x, y)=0$.

An illustrative example follows.

Example 1 Suppose that a particle with mass $m=1$ is moving in a line under the action of two forces. One force possesses a smooth (i.e. of class $C^{\infty}$ ) potential $u$ that depends on the

Fig. 1 Integral curves on the surface defined by (1)

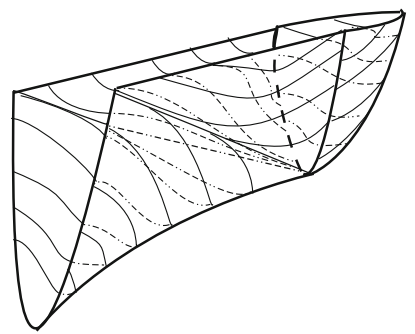


Fig. 2 Projections of the integral curves onto the $(x, y)$-plane

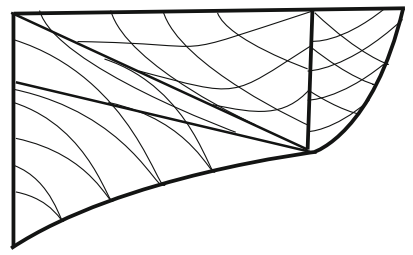

position $x$ of the point on the line. The other is the force of friction, which is proportional to the velocity with proportionality factor $k \geq 0$. That also depends on the position of the point on the line. By Newton second law the equations of the motion is

$$
\ddot{x}=-\dot{u}(x)-k(x) \dot{x} ;
$$

where $x$ is the position, $\dot{x}$ is the velocity and $\ddot{x}$ is the acceleration of the particle. As usual, taking $\dot{x}=y$ and $\ddot{x}=\dot{y}$ we get the planar system

$$
\dot{x}=y, \quad \dot{y}=\ddot{x}=-\dot{u}(x)-k(x) y .
$$

If $k=0$ the system is conservative, and the total energy

$$
E=\frac{y^{2}}{2}+u(x)
$$

is a first integral. It means that the orbits of the system are contained in the level curves of $E$.

For $k>0$ the trajectories in the plane $x, E$ coincide with the family of the integral curves of an implicit differential equation. In fact, differentiating the total energy we get

$$
\dot{E}=-2 k(x)(E-u(x)) \text {. }
$$

Since $y^{2}=2(E-u(x)),\left(\frac{d E}{d x}\right)^{2}=\frac{\dot{E}^{2}}{\dot{x}^{2}}=\frac{\dot{E}^{2}}{y^{2}}$, the trajectories in the plane $x, E$ satisfies

$$
\left(\frac{d E}{d x}\right)^{2}=2 k(x)^{2}(E-u(x))=g(x, E) .
$$

A piecewise implicit equation is obtained considering the above example but with discontinuity in the friction force. More precisely, from a point $x_{\sigma}$ the force of friction has another factor $k_{1}>0$, with $k_{1} \neq k$. Thus our equation becomes

$$
\left(\frac{d E}{d x}\right)^{2}=\left\{\begin{array}{l}
2 k(x)^{2}(E-u(x)) \quad x<x_{\sigma} \\
2 k_{1}(x)^{2}(E-u(x)) \quad x>x_{\sigma}
\end{array}\right.
$$

If we assume that the variable $x$ represents the time then the Eq. (1) corresponds to the system

$$
\dot{x}=1, \quad(\dot{y})^{2}=g(x, y)=\left\{\begin{array}{ll}
g_{1}(x, y) & \varphi(x, y) \geq 0 \\
g_{2}(x, y) & \varphi(x, y) \leq 0
\end{array} .\right.
$$

Our primary interest consists to exhibit conditions for the existence of sliding motion on the discontinuity curve $\varphi(x, y)=0$. 


\subsection{Results}

In what follows we discuss roughly, the main results proved in this article. The precise statements are presented in the Sects. 3 and 5. Take a system like (4) with discontinuity curve given by $\Sigma=\{\varphi(x, y)=0\}$. The curve $\Sigma$ divides the domain $U$ into two sub-regions $\Sigma_{+}=\{\varphi(x, y)>0\}$ and $\Sigma_{-}=\{\varphi(x, y)<0\}$. Associated to the system (4) four possible pairs of vector fields selected from $\frac{\partial}{\partial x} \pm \sqrt{g_{i}} \frac{\partial}{\partial y}, i=1,2$, defined in the regions $\Sigma_{+}$and $\Sigma_{-}$.

(A) If $\frac{\partial \varphi}{\partial x}=0$ ( resp. $\frac{\partial \varphi}{\partial y}=0$ ) we give a complete classification of points on $\Sigma$ as sewing, sliding or escaping for each possible choice of vector fields. See Theorem 1 (resp. Theorem 2 ).

(B) We classify generically the points in the region $\Sigma$ when the vector fields in both sides of $\Sigma$ are those ones presented in Table 1, Sect. 2.2. See Theorems 3 and 4 .

(C) An explicit expression for the sliding system that occurs in the region $\Sigma$ is given for the case $\frac{\partial \varphi}{\partial y} \neq 0$. See Theorem 5 .

(D) Under certain conditions on the transition function, the regularization of discontinuous implicit differential systems provides an implicit singular perturbation. In general this transition can be done explicitly. See Theorem 6.

The paper is organized as follows. In Sects. 2.1, 2.2 we introduce basic notions of implicit differential equations. For more details we refer [1,5,8]. In Sects. 2.3, 2.4 we present discontinuous vector fields and regularization process following the references $[6,9,13,16,17]$. In Sects. 3 and 4 we present and prove our results and in Sect. 5 we introduce the concept of regularization of discontinuous implicit differential systems.

\section{Preliminaries}

In this section we give some definitions about implicit differential equation and discontinuous vector fields.

\subsection{Implicit Differential Equations}

A general implicit differential equation can be obtained in the following way. Let $F: \mathbb{R}^{3} \rightarrow \mathbb{R}$ be a $C^{r}$-function with $r \geq 1$. We assume that 0 is a regular value of $F$. Thus the set $M \subseteq \mathbb{R}^{3}$ given by

$$
M=\left\{(x, y, p) \in \mathbb{R}^{3} ; F(x, y, p)=0\right\}
$$

is a $C^{r}$ - manifold. Moreover, the variable $p$ is the derivative $p=\frac{d y}{d x}$.

Our interest is when the derivative $F_{p}(q)=0$ at some $q \in M$. In fact, for $F_{p}(q) \neq 0$, the implicit function theorem implies that there exist $f(x, y)$ and an open neighborhood $V$ such that for any $(x, y, p) \in V$ we have

$$
F(x, y, p)=0 \Longleftrightarrow p=f(x, y) .
$$

Consider a direction field on the surface $M$ defined as follows. At a point $q_{0}=$ $\left(x_{0}, y_{0}, p_{0}\right) \in M$, consider the plane

$$
C P_{q_{0}}=\left\{\theta=(x, y, p) \in \mathbb{R}^{3}: d y=p_{0} d x\right\} .
$$


Fig. 3 Direction field at $q \in M$

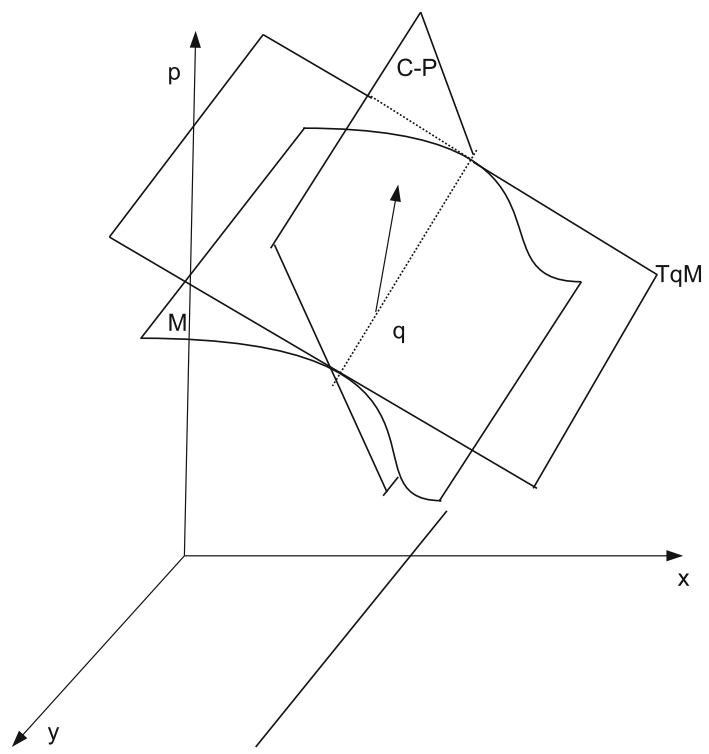

More precisely $\theta \in C P_{q_{0}}$ if its projection onto the $(x, y)$-plane forms an angle with the $x$-axis whose tangent is equal to $p_{0}$. The plane $C P q_{0}$ is called the contact-plane. Assume that $C P_{q_{0}}$ intersects the tangent plane $T_{q_{0}} M$ in a line. Moreover assume for simplicity that the $C P_{q} \cap T_{q} M$ is a line at all $q$ nearby $q_{0}$. Thus we have a direction field on a neighborhood of $q_{0} \in M$ (Fig. 3).

The integral curves of $F(x, y, p)=0$ are the integral curves of this direction field. To solve this equation it is necessary to find these curves.

A direction field, as described above, can be obtained taking the vector field

$$
\xi=F_{p} \frac{\partial}{\partial x}+p F_{p} \frac{\partial}{\partial y}-\left(F_{x}+p F_{y}\right) \frac{\partial}{\partial p} .
$$

The direction of the $p$-axis in the space $\mathbb{R}^{3}$ is called vertical direction and the projection

$$
\pi: M \rightarrow \mathbb{R}^{2}, \quad \pi(x, y, p)=(x, y)
$$

is the vertical projection.

A point $q \in M$ is said to be regular if it is not a critical point of $\pi$. In other words, a point of $M$ is regular if the tangent plane at this point is not vertical. The other points of the surface $M$ are said singular (the points such that $F(q)=F_{p}(q)=0$ ). The set of singular points, $\mathcal{C}$, is called criminant of $M$ and its image, $\mathcal{D}$, via the application $\pi$, is called the discriminant. Note that if $q \in \mathcal{C}$ then $F(q)=F_{p}(q)=0$. If $F_{p p}(q) \neq 0$ then $q$ is a fold point of $F$, and if $F_{p p}(q)=0$ and $F_{p p p}(q) \neq 0 q$ it is a cusp point of $F$.

Example 2 Consider the differential equation $p^{2}=x$. In this case the surface $M$ is a parabolic cylinder (see 5). The discriminant curve is the $y$-axis. In order to find the integral curves, we write down the conditions for $d x, d y$ and $d p$ at the point $q=(x, y, p)$ of the surface $M$ :

$$
\begin{cases}p^{2}=x, & \text { the condition } q \in M \\ 2 p d p=d x, & \text { the condition of tangence to } M \\ d y=p d x, & \text { the condition of the contact plane }\end{cases}
$$


Fig. 4 Integral curves on $M$

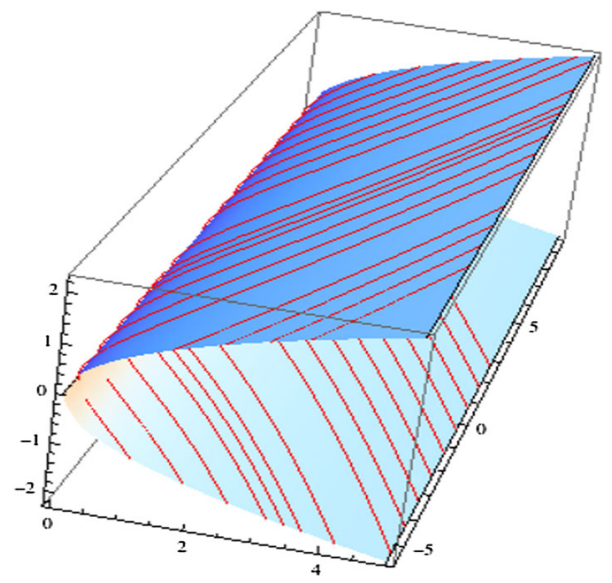

Fig. 5 Projections of the integral curves onto the $(x, y)$-plane

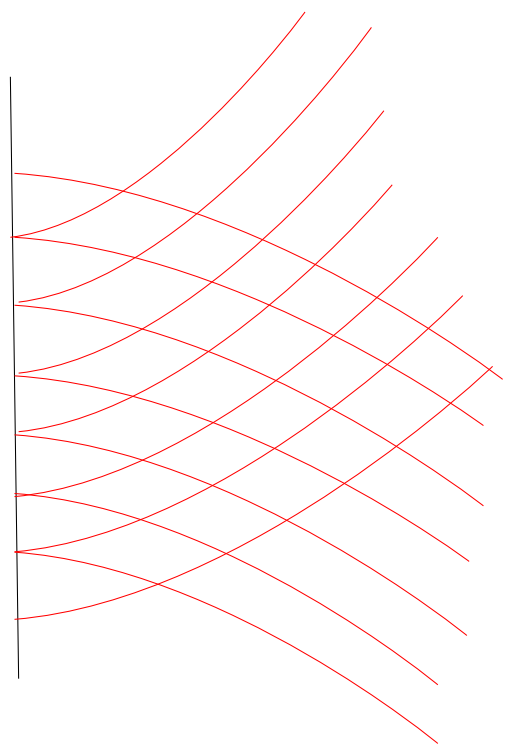

Consequently, in coordinates $(p, y)$, the integral curves are determined from the equation $d y=2 p^{2} d p$.

Hence, the integral curves on $M$ are given by the relations $y+C=\frac{2}{3} p^{3}, x=p^{2}$ (see Fig. 4 ). Their projections onto the ( $x, y)$-plane are semicubical parabolas (see Fig. 5).

\subsection{Implicit Ordinary Differential Equations with Degree 2}

Now we consider

$$
F(x, y, p)=p^{2}-g(x, y)=0
$$

where $g(x, y)$ is a $C^{1}$ real function satisfying $g(0,0)=0$. 
Table 1 Generic singularities of first order implicit differential systems

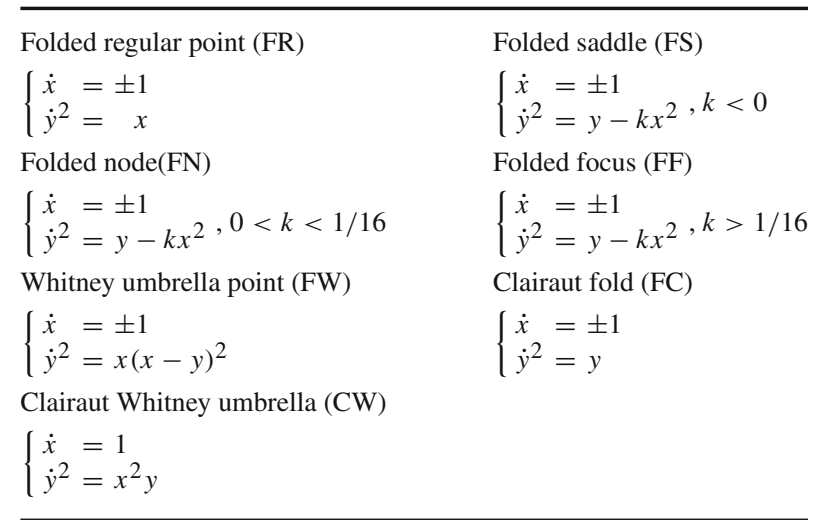

Since $F_{p p}(q)=2$ for all $q \in \mathcal{C}$ we derive that $q$ is a fold point of $F$. More

$$
\xi(q)=F_{p} \frac{\partial}{\partial x}+p F_{p} \frac{\partial}{\partial y}-\left(F_{x}+p F_{y}\right) \frac{\partial}{\partial p}=-F_{x} \frac{\partial}{\partial p}
$$

provided $q \in \mathcal{C}$.

The points $q \in \mathcal{C} \subset M$ are classified as follows:

(a) $q$ is a folded regular point if $F_{x}(q) \neq 0$.

(b) $q$ is a folded singular if $F_{x}(q)=0$.

Since $\xi(q)=-F_{x} \frac{\partial}{\partial p}$ on $\mathcal{C}$ all folded singular on $\mathcal{C}$ are singular points of $\xi$.

We say that $q$ is a folded saddle point (resp. folded node, folded focus points) if $q$ is a saddle singularity (resp. node, focus singularities) of $\Pi \circ \xi$ where $\Pi(x, y, p)=(x, p)$. It is easy to see that if $q \in \mathcal{C}$ with $F_{y}(q) \neq 0$ then:

- $F_{x x}(q)>0 \Rightarrow q$ is folded saddle ;

- $F_{x x}(q)<0$ and $F_{x x}(q)>-\frac{1}{8}\left(F_{y}(q)\right)^{2} \Rightarrow q$ is folded node and

- $F_{x x}(q)<0$ and $F_{x x}(q)<-\frac{1}{8}\left(F_{y}(q)\right)^{2} \Rightarrow q$ is folded focus.

We say that $q \in \mathcal{C}$ is a Clairaut fold if $F_{y}(q) \neq 0$ and $F_{x x}(q)=0$.

The case where a folded singular point $q \in \mathcal{C}$ satisfies $F_{y}(q)=0$ is too degenerate. In [8] the authors present a complete list of generic singularities of first order implicit systems on the plane up to smooth orbital equivalence. See Table (1). Two germs of system surfaces are called orbitally equivalent if there exists a germ of diffeomorphism which maps any phase curve of one system to a phase curve of another system.

\subsection{Discontinuous Vector Fields}

Consider an open subset $U \subset \mathbb{R}^{2}$ and let $\varphi: U \rightarrow \mathbb{R}$ be a $C^{\infty}$ function having $0 \in \mathbb{R}$ as a regular value for any $q \in \Sigma=\varphi^{-1}(0)$. We denote $\Lambda$ the set of vector fields $X=\left(X_{1}, X_{2}\right)$ such that

$$
X(q)=\left\{\begin{array}{l}
X_{1}(q), \text { if } q \in \Sigma^{+}=\{\varphi(q) \geq 0\} \\
X_{2}(q), \text { if } q \in \Sigma^{-}=\{\varphi(q) \leq 0\}
\end{array}\right.
$$

where $X_{1}, X_{2}: U \rightarrow \mathbb{R}^{2}$ are smooth vector fields defined on $U$. The topology considered in $\Lambda$ is the usual product topology. For details see $[6,9,14]$. 
Fig. 6 Sliding vector field

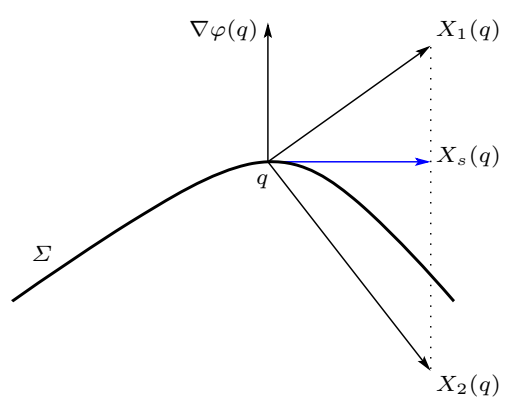

We say that $q \in \Sigma$ is regular if both $X_{1}$ and $X_{2}$, cross $\Sigma$ at $q$ otherwise $q$ is said singular. Following the terminology established in [11], the regular points in $\Sigma$ are classified as:

- Sliding region (both point to $\Sigma$ ):

$$
\Sigma_{s l}=\left\{X_{1} \varphi<0, X_{2} \varphi>0\right\}
$$

- Escaping region (both leave $\Sigma$ ):

$$
\Sigma_{e s}=\left\{X_{1} \varphi>0, X_{2} \varphi<0\right\}
$$

- Sewing region (one point to $\Sigma$ and the other leaves $\Sigma$ ):

$$
\Sigma_{s w}=\left\{\left(X_{1} \varphi\right)\left(X_{2} \varphi\right)>0\right\} .
$$

On $\Sigma_{s}=\Sigma_{s l} \cup \Sigma_{e s}$ the flow slides and follows a well defined smooth vector field $X_{s}$ called sliding vector field. We define $X_{s}(p)$ as the tangent vector defined on $\Sigma$ and contained in the cone generated by $X_{1}$ and $X_{2}$ (Fig. 6).

We say that a point $q \in \Sigma$ is fold point of $X \in \Lambda$ if $X \varphi=0$ and $X^{2} \varphi \neq 0$. We say that $q \in \Sigma$ is a singular point of $X$ if $q$ is a fold point of $X_{1}$ or $q$ is fold point of $X_{2}$ or $\left(X_{1} \varphi\right)\left(X_{2} \varphi\right)<0$ and $\operatorname{det}\left[X_{1}, X_{2}\right]=0$.

An important tool in the study of non-smooth dynamical system is the $\psi$-regularization process. A $\mathbb{C}^{\infty}$ function $\psi: \mathbb{R} \rightarrow \mathbb{R}$ is a transition function if $\psi(t)=-1$ for $t \leq-1$, $\psi(t)=1$ for $t \geq 1$ and $\psi^{\prime}(t)>0$ if $t \in(-1,1)$. The $\psi$-regularization of $X=\left(X_{1}, X_{2}\right) \in$ $\Lambda$, is the one-parameter family $X_{\varepsilon}$ given by

$$
X_{\varepsilon}=\left(\frac{1}{2}+\frac{\psi_{\varepsilon}(\varphi)}{2}\right) X_{1}+\left(\frac{1}{2}-\frac{\psi_{\varepsilon}(\varphi)}{2}\right) X_{2}
$$

with $\psi_{\varepsilon}(t)=\psi\left(\frac{t}{\varepsilon}\right)$, for $\varepsilon>0$. Note that $X_{\varepsilon}$ is equal to $X_{1}$ in all points of $\Sigma^{+}$whose distance to $\Sigma$ is bigger than $\varepsilon$ and $X_{\varepsilon}$ is equal to $X_{2}$ in all points of $\Sigma^{-}$whose distance to $\Sigma$ is bigger than $\varepsilon$. For details see [13,16,17]. A description of some qualitative and geometric aspects of non-smooth dynamical systems theory around typical singularities can be found in [15].

\subsection{Implicit Differential Systems with Discontinuity and the Set $\Omega$}

Let $V \in \mathbb{R}^{2}$ be an open neighborhood of $(0,0)$. Consider $g_{i}, i=1,2$, be real functions of class $C^{1}$ in $V$ with $g_{i}(0,0)=0$ and the implicit differential system:

$$
S_{i}(x, y, \dot{x}, \dot{y})=\left(\dot{x}-1, \dot{y}^{2}-g_{i}(x, y)\right)=(0,0), \quad i=1,2 ;
$$

with $(x, y)$ in $U_{i} \subseteq V$ given by $U_{i}=\left\{(x, y) \in \mathbb{R}^{2}: g_{i}(x, y)>0\right\}$. 


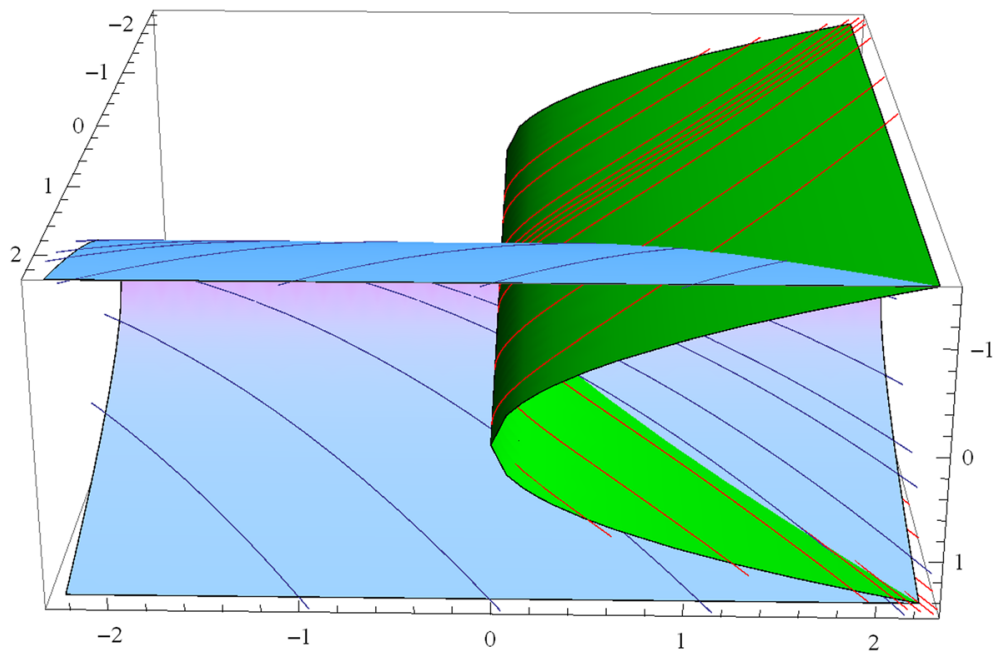

Fig. 7 The surfaces $(\dot{y})^{2}-x=0$ and $(\dot{y})^{2}-y=0$

Let $M_{1}$ and $M_{2}$ be the surfaces defined by $(\dot{y})^{2}-g_{1}(x, y)=0,(\dot{y})^{2}-g_{2}(x, y)=0$ respectively in the $(x, y, \dot{y})$-space. Consider $T=M_{1} \cap M_{2}$ and $U=U_{1} \cap U_{2} \neq \emptyset$. If $T \neq \emptyset$ we denote by $S=\left(S_{1}, S_{2}\right) \in \Omega$ the system defined by

$$
\left\{\begin{array}{l}
S_{1}(x, y, \dot{x}, \dot{y})=(0,0), \text { if } \varphi(x, y)=g_{1}(x, y)-g_{2}(x, y) \geq 0 \\
S_{2}(x, y, \dot{x}, \dot{y})=(0,0), \text { if } \varphi(x, y)=g_{1}(x, y)-g_{2}(x, y) \leq 0
\end{array} .\right.
$$

Note that $S_{1}$ and $S_{2}$ define four non-smooth vector fields $\left(V_{1 \alpha}, V_{2 \beta}\right) \in \Lambda, \alpha, \beta=\{+,-\}$ given by

$$
V_{i,+}(x, y)=\frac{\partial}{\partial x}+\sqrt{g_{i}(x, y)} \frac{\partial}{\partial y}, \quad(x, y) \in U_{i}
$$

and

$$
V_{i,-}(x, y)=\frac{\partial}{\partial x}-\sqrt{g_{i}(x, y)} \frac{\partial}{\partial y}, \quad(x, y) \in U_{i} .
$$

Example 3 Consider the implicit differential equations

$$
S_{1}(x, y, \dot{x}, \dot{y})=\left(\dot{x}-1, \dot{y}^{2}-x\right)=(0,0)
$$

with $(x, y) \in \mathbb{R}^{2}$ satisfying that $x \geq 0$; and

$$
S_{2}(x, y, \dot{x}, \dot{y})=\left(\dot{x}-1, \dot{y}^{2}-y\right)=(0,0)
$$

with $(x, y) \in \mathbb{R}^{2}$ satisfying that $y \geq 0$.

Now we take the discontinuous system

$$
S(x, y, \dot{x}, \dot{y})=\left\{\begin{array}{l}
S_{1}(x, y, \dot{x}, \dot{y})=\{0,0\}, \text { if } \varphi(x, y) \geq 0 \\
S_{2}(x, y, \dot{x}, \dot{y})=\{0,0\}, \text { if } \varphi(x, y) \leq 0
\end{array}\right.
$$

where $\varphi(x, y)=x-y$ and $\Sigma=\varphi^{-1}(0)$ (see Fig. 7).

Since a discontinuous implicit differential system (DIDS) define four non-smooth vector fields we have that $q \in \Sigma$ can be a sewing point for a choice $\alpha, \beta$ and a sliding point point 
Table 2 Sewing, sliding and escaping regions for the $\left(S_{1}, S_{2}\right) \in \Omega$ with $\frac{\partial \varphi}{\partial x}(x, y)=0$

\begin{tabular}{lll}
\hline$\Lambda$ & $\Sigma_{s w}$ & $\Sigma_{s l} \cup \Sigma_{e s}$ \\
\hline$\left(V_{1+}, V_{2+}\right)$ & $\Sigma$ & $\emptyset$ \\
$\left(V_{1+}, V_{2-}\right)$ & $\emptyset$ & $\Sigma$ \\
$\left(V_{1-}, V_{2+}\right)$ & $\emptyset$ & $\Sigma$ \\
$\left(V_{1-}, V_{2-}\right)$ & $\Sigma$ & $\emptyset$
\end{tabular}

for another. We found the sliding and sewing regions on $\Sigma$ evaluating the sign of the Lie derivative

$$
\left[V_{i,+} . \varphi\right]=\frac{\partial \varphi}{\partial x}+\frac{\partial \varphi}{\partial y} \sqrt{g_{i}} \text { and }\left[V_{i,-} . \varphi\right]=\frac{\partial \varphi}{\partial x}-\frac{\partial \varphi}{\partial y} \sqrt{g_{i}}
$$

\section{Statement of the Main Results}

In this section we state our main results. As before we denote $S=\left(S_{1}, S_{2}\right) \in \Omega$ a pair like (11).

Theorem 1 If $S=\left(S_{1}, S_{2}\right) \in \Omega$ satisfies that $\frac{\partial \varphi}{\partial x}(x, y)=0$, for any $(x, y) \in U$, then the regular points in $\Sigma$ are classified as shown in Table 2.

If $S=\left(S_{1}, S_{2}\right) \in \Omega$ with $S_{1} \neq S_{2}$ and $S_{1}, S_{2} \in\{F F, F N, F S, F C\}$, (see Table 1) then $\left(S_{1}, S_{2}\right)$ satisfies the hypotheses of Theorem 1 .

Theorem 2 If $S=\left(S_{1}, S_{2}\right) \in \Omega$ satisfies that $\frac{\partial \varphi}{\partial y}(x, y)=0$, for any $(x, y) \in U$, then $\Sigma=\Sigma_{\text {sw }}$ for all $\left(V_{1 i}, V_{2 j}\right) \in \Lambda$ with $i, j \in\{+,-\}$.

Next theorem analyses pairs of implicit systems FR, FS, FN and FF of described in Table 1. We denote $M_{i}=\left\{p^{2}-g_{i}(x, y)=0\right\}, i=1,2$ and $\pi: \mathbb{R}^{3} \rightarrow \mathbb{R}^{2}$ is the projection $\pi(x, y, p)=(x, y)$.

Theorem 3 Consider $\left(S_{1}, S_{2}\right)=(F R, t) \in \Omega$, where $t \in I=\{F S, F N, F F\}$. Assuming $\Sigma=\pi\left(M_{1} \cap M_{2}\right)$ we have that $\Sigma=\bar{\Sigma}_{1} \cup \bar{\Sigma}_{2} \cup \bar{\Sigma}_{3}$ for $k<\frac{1}{8}$ where

$$
\begin{aligned}
& \Sigma_{1}=\left\{\left(x, x+k x^{2}\right): x<\frac{1-\sqrt{1-8 k}-4 k}{8 k^{2}}\right\} ; \\
& \Sigma_{2}=\left\{\left(x, x+k x^{2}\right): \frac{1-\sqrt{1-8 k}-4 k}{8 k^{2}}<x<\frac{1+\sqrt{1-8 k}-4 k}{8 k^{2}}\right\} ; \\
& \Sigma_{3}=\left\{\left(x, x+k x^{2}\right): x>\frac{1+\sqrt{1-8 k}-4 k}{8 k^{2}}\right\} .
\end{aligned}
$$

Moreover the regular points in $\Sigma$ for the cases $t=F S, F N, F F$ are classified as shown in Tables 3, 4 and 5 respectively.

Theorem 3 is proved in Sect. 4 .

Theorem 4 Consider $\left(S_{1}, S_{2}\right)=(F R, t) \in \Omega$ and denote $h_{F C}(x)=x$ and $_{C W}(x)=1 / x$. If $\Sigma=\pi\left(M_{1} \cap M_{2}\right)$ then $\Sigma=\bar{\Sigma}_{1} \cup \bar{\Sigma}_{2}$ with 
Table 3 Sewing, sliding and escaping regions for the $(F R, F S)$ system

Table 4 Sewing, sliding and escaping regions for the $(F R, F N)$ system

\begin{tabular}{llll}
\hline$\Lambda$ & $\Sigma_{s w}$ & $\Sigma_{s l}$ & $\Sigma_{\text {es }}$ \\
\hline$\left(V_{1+}, V_{2+}\right)$ & $\Sigma$ & $\emptyset$ & $\emptyset$ \\
$\left(V_{1+}, V_{2-}\right)$ & $\Sigma_{1} \cup \Sigma_{3}$ & $\Sigma_{2}$ & $\emptyset$ \\
$\left(V_{1-}, V_{2+}\right)$ & $\Sigma_{1} \cup \Sigma_{3}$ & $\emptyset$ & $\Sigma_{2}$ \\
$\left(V_{1-}, V_{2-}\right)$ & $\Sigma$ & $\emptyset$ & $\emptyset$ \\
\hline
\end{tabular}

\begin{tabular}{llll}
\hline$\Lambda$ & $\Sigma_{s w}$ & $\Sigma_{s l}$ & $\Sigma_{\text {es }}$ \\
\hline$\left(V_{1+}, V_{2+}\right)$ & $\Sigma$ & $\emptyset$ & $\emptyset$ \\
$\left(V_{1+}, V_{2-}\right)$ & $\Sigma_{1}$ & $\Sigma_{2} \cup \overline{\Sigma_{3}}$ & $\emptyset$ \\
$\left(V_{1-}, V_{2+}\right)$ & $\Sigma_{1}$ & $\emptyset$ & $\Sigma_{2} \cup \overline{\Sigma_{3}}$ \\
$\left(V_{1-}, V_{2-}\right)$ & $\Sigma$ & $\emptyset$ & $\emptyset$ \\
\hline
\end{tabular}

Table 5 Sewing, sliding and escaping regions for the $(F R, F F)$ system

\begin{tabular}{lllll}
\hline$\Lambda$ & $k$ & $\Sigma_{s w}$ & $\Sigma_{s l}$ & $\Sigma_{e s}$ \\
\hline$\left(V_{1+}, V_{2+}\right)$ & $\frac{1}{16}<k<\frac{1}{8}$ & $\Sigma$ & $\emptyset$ & $\emptyset$ \\
$\left(V_{1+}, V_{2-}\right)$ & & $\Sigma_{1}$ & $\Sigma_{2} \cup \overline{\Sigma_{3}}$ & $\emptyset$ \\
$\left(V_{1-}, V_{2+}\right)$ & & $\Sigma_{1}$ & $\emptyset$ & $\Sigma_{2} \cup \overline{\Sigma_{3}}$ \\
$\left(V_{1-}, V_{2-}\right)$ & $\Sigma$ & $\emptyset$ & $\emptyset$ \\
$\left(V_{1 i}, V_{2 j}\right), i, j \in\{+,-\}$ & $k>\frac{1}{8}$ & $\Sigma$ & $\emptyset$ & $\emptyset$ \\
\hline
\end{tabular}

(a)

$$
\begin{aligned}
& \Sigma_{1}=\left\{\left(x, h_{t}(x)\right) \in \Sigma: x<1\right\}, \\
& \Sigma_{2}=\left\{\left(x, h_{t}(x)\right) \in \Sigma: x>1\right\},
\end{aligned}
$$

(b)

for the case $t \in I=\{F C, C W\}$ and

$$
\begin{aligned}
& \Sigma_{1}=\{(x, x+1) \in \Sigma: x<1\} \cup\{(x, x-1) \in \Sigma: x<1\}, \\
& \Sigma_{2}=\{(x, x+1) \in \Sigma: x>1\} \cup\{(x, x-1) \in \Sigma: x>1\},
\end{aligned}
$$

for the case $t=F W$. Moreover the regular points in $\Sigma$ for the cases $t=F C, F W, C W$ are classified as shown in Table 6.

Theorem 4 is proved in Sect. 4.

Proposition 1 Consider $t \in\{A, B\}$ where $A=\left(S_{1}, S_{2}\right), B=\left(S_{2}, S_{1}\right) \in \Omega$ and $\Sigma_{s l}^{t}, \Sigma_{e s}^{t}$ and $\Sigma_{s w}^{t}$ sewing, sliding and escaping regions for the t-system. Then $\Sigma_{s l}^{A}=\Sigma_{e s}^{B}, \Sigma_{e s}^{A}=\Sigma_{s l}^{B}$ and $\Sigma_{s w}^{A}=\Sigma_{s w}^{B}$ for all vector fields $\left(V_{1 i}, V_{2 j}\right) \in \Lambda$ with $i, j \in\{+,-\}$.

Theorem 5 Consider $S=\left(S_{1}, S_{2}\right) \in \Omega$. If $\frac{\partial \varphi}{\partial y}(x, y) \neq 0$, for any $(x, y) \in \Sigma_{s}=\Sigma_{s l} \cup \Sigma_{e s}$ then the sliding vector fields is

$$
\left(V_{1+}, V_{2-}\right)_{s}=\left(V_{1-}, V_{2+}\right)_{s}=\frac{\partial}{\partial x}-\left(\frac{\frac{\partial \varphi}{\partial x}(x, y)}{\frac{\partial \varphi}{\partial y}(x, y)}\right) \frac{\partial}{\partial y} .
$$


Table 6 Sewing, sliding and escaping regions for the $(F R, t)$ systems, where $t \in I=\{F C, F W, C W\}$

\begin{tabular}{llll}
\hline$\Lambda$ & $\Sigma_{s w}$ & $\Sigma_{s l}$ & $\Sigma_{e s}$ \\
\hline$\left(V_{1+}, V_{2+}\right)$ & $\Sigma$ & $\emptyset$ & $\emptyset$ \\
$\left(V_{1+}, V_{2-}\right)$ & $\Sigma_{1}$ & $\Sigma_{2}$ & $\emptyset$ \\
$\left(V_{1-}, V_{2+}\right)$ & $\Sigma_{1}$ & $\emptyset$ & $\Sigma_{2}$ \\
$\left(V_{1-}, V_{2-}\right)$ & $\Sigma$ & $\emptyset$ & $\emptyset$
\end{tabular}

\section{Proofs of the Main Results}

Proof of Theorem 1 Consider the discontinuous system $\left(S_{1}, S_{2}\right) \in \Omega$, if $\frac{\partial \varphi}{\partial x}(x, y)=0$. The sign of the Lie derivative determines the regions on $\Sigma$. We have $\left[V_{\ell,+} . \varphi\right]=\frac{\partial \varphi}{\partial y} \sqrt{g_{\ell}}$ and $\left[V_{\ell,-} . \varphi\right]=-\frac{\partial \varphi}{\partial y} \sqrt{g_{\ell}}$. If $(i, j)=(+,+)$ or $(i, j)=(-,-)$ then $\left[V_{1, i} . \varphi\right]\left[V_{2, j} . \varphi\right]>0$ for all $(x, y) \in \Sigma$ and we conclude that $\Sigma_{s w}=\Sigma, \Sigma_{s l}=\emptyset$ and $\Sigma_{e s}=\emptyset$. From the other hand, if $(i, j)=(+,-)$ or $(i, j)=(-,+)$ then $\left[V_{1, i} . \varphi\right]\left[V_{2, j} . \varphi\right]<0$ for all $(x, y) \in \Sigma$ and thus $\Sigma_{s w}=\emptyset, \Sigma_{s l} \cup \Sigma_{e s}=\Sigma$.

Example $4 S=\left(S_{1}, S_{2}\right) \in \Omega$ with $g_{1}(x, y)=x$ and $g_{2}(x, y)=-x+3$ satisfies the hypothesis of Theorem 2 .

Proof of Theorem 3 Consider the discontinuous system $S=\left(S_{1}, S_{2}\right)$ :

$$
S=\left\{\begin{aligned}
\left(\dot{x}-1, \dot{y}^{2}-x\right) & =(0,0), \text { if } \varphi(x, y) \geq 0 \\
\left(\dot{x}-1, \dot{y}^{2}-y+k x^{2}\right) & =(0,0), \text { if } \varphi(x, y) \leq 0
\end{aligned}\right.
$$

where $\varphi(x, y)=x-y+k x^{2}$. The switching curve is given by $\Sigma=\varphi^{-1}(0) \cap U=\{(x, y) \in$ $\left.\mathbb{R}^{2} ; y=x+k x^{2}\right\}$. As before, four vector fields are determined:

$$
\begin{aligned}
& V_{1+}(x, y)=\frac{\partial}{\partial x}+\sqrt{x} \frac{\partial}{\partial y}, \\
& V_{1-}(x, y)=\frac{\partial}{\partial x}-\sqrt{x} \frac{\partial}{\partial y}, \\
& V_{2+}(x, y)=\frac{\partial}{\partial x}+\sqrt{y-k x^{2}} \frac{\partial}{\partial y}, \\
& V_{2-}(x, y)=\frac{\partial}{\partial x}-\sqrt{y-k x^{2}} \frac{\partial}{\partial y} .
\end{aligned}
$$

The sign of the Lie derivative $V_{i} . \varphi$, with $i \in\{1,2\}$ and $(x, y) \in \Sigma$ determines the regions on $\Sigma$. Note that if $(x, y) \in \Sigma$ then $(x, y)=\left(x, x+k x^{2}\right)$. We have $\left(V_{1,+}\right) \cdot \varphi=2 k x+1-\sqrt{x}$, $\left(V_{1,-}\right) \cdot \varphi=2 k x+1+\sqrt{x},\left(V_{2,+}\right) \cdot \varphi=2 k x+1-\sqrt{x}$ and $\left(V_{2,-}\right) \cdot \varphi=2 k x+1+\sqrt{x}$. Note if $k<0$ we have

(a) If $i=+, j=+$ then $\left[V_{1, i} . \varphi\right]\left[V_{2, j} . \varphi\right]>0$ for all $(x, y) \in \Sigma$. Therefore $\Sigma_{s w}=$ $\Sigma, \Sigma_{s l}=\emptyset$ and $\Sigma_{e s}=\emptyset$.

(b) If $i=+, j=-$ then $\left[V_{1, i} \cdot \varphi\right]\left[V_{2, j} . \varphi\right]>0$ for all $(x, y) \in \Sigma_{1} \cup \Sigma_{3}$ and $\left[V_{1, i} . \varphi\right]\left[V_{2, j} . \varphi\right]<0$ and $\left[V_{1, i} . \varphi\right]<0$ for all $(x, y) \in \Sigma_{2}$. Therefore $\Sigma_{s w}=$ $\Sigma_{1} \cup \Sigma_{3}, \Sigma_{s l}=\Sigma_{2}$ and $\Sigma_{e s}=\emptyset$.

(c) If $i=-, j=+$ then $\left[V_{1, i} \cdot \varphi\right]\left[V_{2, j} \cdot \varphi\right]>0$ for all $(x, y) \in \Sigma_{1} \cup \Sigma_{3}$ and $\left[V_{1, i} . \varphi\right]\left[V_{2, j} . \varphi\right]<0$ and $\left[V_{2, j} . \varphi\right]<0$ for all $(x, y) \in \Sigma_{2}$. Therefore $\Sigma_{s w}=$ $\Sigma_{1} \cup \Sigma_{3}, \Sigma_{s l}=\emptyset$ and $\Sigma_{e s}=\Sigma_{2}$. 
Fig. $8 V_{1, \pm}=\frac{\partial}{\partial x} \pm \sqrt{x} \frac{\partial}{\partial y}$ and

$V_{2, \pm}=\frac{\partial}{\partial x} \pm \sqrt{y-k x^{2}} \frac{\partial}{\partial y}$,

with $k<0$
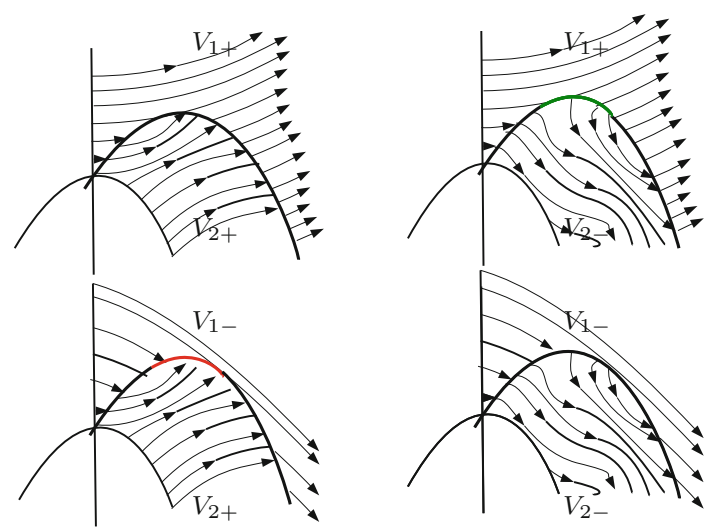

(d) If $i=-, j=-$ then $\left[V_{1, i} \cdot \varphi\right]\left[V_{2, j} \cdot \varphi\right]>0$ for all $(x, y) \in \Sigma$. Therefore $\Sigma_{s w}=$ $\Sigma, \Sigma_{s l}=\emptyset$ and $\Sigma_{e s}=\emptyset$.

Therefore, we conclude the veracity of the data presented in Table 3. See Fig. 8.

If $0<k<\frac{1}{8}$ we have

(a) If $i=+, j=+$ then $\left[V_{1, i} \cdot \varphi\right]\left[V_{2, j} \cdot \varphi\right]>0$ for all $(x, y) \in \Sigma$. Therefore $\Sigma_{s w}=$ $\Sigma, \Sigma_{s l}=\emptyset$ and $\Sigma_{e s}=\emptyset$.

(b) If $i=+, j=-$ then $\left[V_{1, i} \cdot \varphi\right]\left[V_{2, j} . \varphi\right]>0$ for all $(x, y) \in \Sigma_{1}$ and $\left[V_{1, i} \cdot \varphi\right]\left[V_{2, j} . \varphi\right]<0$ and $\left[V_{1, i} . \varphi\right]<0$ for all $(x, y) \in \Sigma_{2} \cup \overline{\Sigma_{3}}$. Therefore $\Sigma_{s w}=\Sigma_{1}, \Sigma_{s l}=\Sigma_{2} \cup \overline{\Sigma_{3}}$ and $\Sigma_{e s}=\emptyset$.

(c) If $i=-, j=+$ then $\left[V_{1, i} . \varphi\right]\left[V_{2, j} . \varphi\right]>0$ for all $(x, y) \in \Sigma_{1}$ and $\left[V_{1, i} . \varphi\right]\left[V_{2, j} . \varphi\right]<0$ and $\left[V_{2, j} . \varphi\right]<0$ for all $(x, y) \in \Sigma_{2} \cup \overline{\Sigma_{3}}$. Therefore $\Sigma_{s w}=\Sigma_{1}, \Sigma_{s l}=\varnothing$ and $\Sigma_{e s}=\Sigma_{2} \cup \overline{\Sigma_{3}}$.

(d) If $i=-, j=-$ then $\left[V_{1, i} \cdot \varphi\right]\left[V_{2, j} \cdot \varphi\right]>0$ for all $(x, y) \in \Sigma$. Therefore $\Sigma_{s w}=$ $\Sigma, \Sigma_{s l}=\emptyset$ and $\Sigma_{e s}=\emptyset$.

Therefore, we conclude the veracity of the data presented in Tables 4 and 5 until the fourth line (see Fig. 9).

If $k>\frac{1}{8}$ we have if $i, j \in\{+,-\}$ then $\left[V_{1, i} . \varphi\right]\left[V_{2, j} . \varphi\right]>0$ for all $(x, y) \in \Sigma$. Therefore $\Sigma_{s w}=\Sigma, \Sigma_{s l}=\emptyset$ and $\Sigma_{e s}=\emptyset$. So we conclude the veracity of the data presented in Table 5 (see Fig. 10).

Proof of Theorem 4 Consider the discontinuous system $\left(F_{1}, t\right)$. If $t=F C$ we have

$$
\left(S_{1}, S_{2}\right)=\left\{\begin{array}{l}
\left(\dot{x}-1, \dot{y}^{2}-x\right)=(0,0), \text { if } \varphi(x, y) \geq 0 \\
\left(\dot{x}-1, \dot{y}^{2}-y\right)=(0,0), \text { if } \varphi(x, y) \leq 0
\end{array}\right.
$$

where $\varphi(x, y)=x-y$. The switching curve is given by $\Sigma=\varphi^{-1}(0) \cap U=\{(x, y) \in$ $\left.\mathbb{R}^{2} ; y=x\right\}$. As before, we have four vector fields determined by the implicit differential equations:

$$
\begin{aligned}
& V_{1+}(x, y)=\frac{\partial}{\partial x}+\sqrt{x} \frac{\partial}{\partial y}, \\
& V_{1-}(x, y)=\frac{\partial}{\partial x}-\sqrt{x} \frac{\partial}{\partial y},
\end{aligned}
$$


Fig. $9 V_{1, \pm}=\frac{\partial}{\partial x} \pm \sqrt{x} \frac{\partial}{\partial y}$ and $V_{2, \pm}=\frac{\partial}{\partial x} \pm \sqrt{y-k x^{2}} \frac{\partial}{\partial y}$, with $0<k<1 / 8$
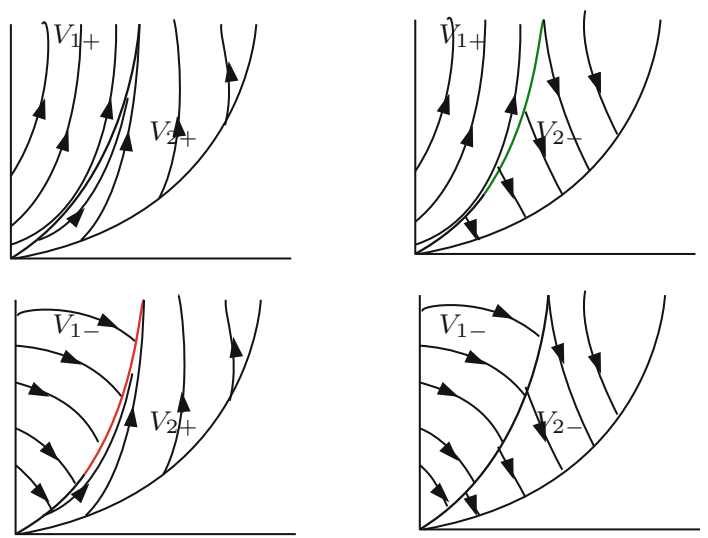

Fig. $10 V_{1, \pm}=\frac{\partial}{\partial x} \pm \sqrt{x} \frac{\partial}{\partial y}$

and $V_{2, \pm}=\frac{\partial}{\partial x} \pm \sqrt{y-k x^{2}} \frac{\partial}{\partial y}$, with $k \geq 1 / 8$
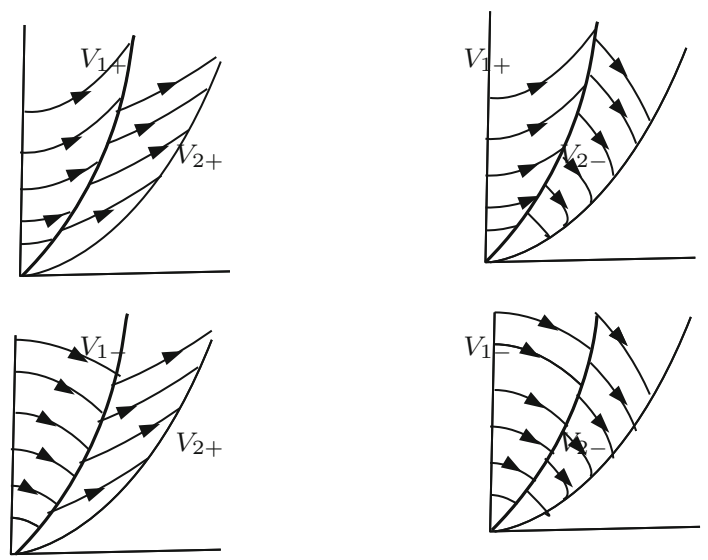

$$
\begin{aligned}
& V_{2+}(x, y)=\frac{\partial}{\partial x}+\sqrt{y} \frac{\partial}{\partial y}, \\
& V_{2-}(x, y)=\frac{\partial}{\partial x}-\sqrt{y} \frac{\partial}{\partial y} .
\end{aligned}
$$

The signs of the Lie derivative $V_{i} . \varphi, i \in\{1,2\}$ and $(x, y) \in \Sigma$ determine the sliding and sewing the regions on $\Sigma$. Note that if $\left(x, h_{t}(x)\right) \in \Sigma$ then $\left(x, h_{t}(x)\right)=(x, x)$. We have $\left(V_{1,+}\right) \cdot \varphi=\left(V_{2,+}\right) \cdot \varphi=1-\sqrt{x}$ and $\left(V_{1,-}\right) \cdot \varphi=\left(V_{2,-}\right) \cdot \varphi=1+\sqrt{x}$.

(a) If $i=+, j=+$ then $\left[V_{1, i} \cdot \varphi\right]\left[V_{2, j} \cdot \varphi\right]>0$ for all $(x, y) \in \Sigma$. Therefore $\Sigma_{s w}=$ $\Sigma, \Sigma_{s l}=\emptyset$ and $\Sigma_{e s}=\emptyset$.

(b) If $i=+, j=-$ then $\left[V_{1, i} . \varphi\right]\left[V_{2, j} . \varphi\right]>0$ for all $(x, y) \in \Sigma_{1}$ and $\left[V_{1, i} . \varphi\right]\left[V_{2, j} . \varphi\right]<0$ and $\left[V_{1, i} . \varphi\right]<0$ for all $(x, y) \in \Sigma_{2}$. Therefore $\Sigma_{s w}=\Sigma_{1}, \Sigma_{s l}=\Sigma_{2}$ and $\Sigma_{e s}=\emptyset$.

(c) If $i=-, j=+$ then $\left[V_{1, i} . \varphi\right]\left[V_{2, j} . \varphi\right]>0$ for all $(x, y) \in \Sigma_{1}$ and $\left[V_{1, i} . \varphi\right]\left[V_{2, j} . \varphi\right]<0$ and $\left[V_{2, j} . \varphi\right]<0$ for all $(x, y) \in \Sigma_{2}$. Therefore $\Sigma_{s w}=\Sigma_{1}, \Sigma_{s l}=\emptyset$ and $\Sigma_{e s}=\Sigma_{2}$.

(d) If $i=-, j=-$ then $\left[V_{1, i} \cdot \varphi\right]\left[V_{2, j} \cdot \varphi\right]>0$ for all $(x, y) \in \Sigma$. Therefore $\Sigma_{s w}=$ $\Sigma, \Sigma_{s l}=\emptyset$ and $\Sigma_{e s}=\emptyset$.

Therefore, the veracity of the data presented in Table 6 is proved for case $t=F C$. 
If $t=C W$ Consider the discontinuous system $S=\left(S_{1}, S_{2}\right)$ :

$$
S=\left\{\begin{aligned}
\left(\dot{x}-1, \dot{y}^{2}-x\right) & =(0,0), \text { if } \varphi(x, y) \geq 0 \\
\left(\dot{x}-1, \dot{y}^{2}-y x^{2}\right) & =(0,0), \text { if } \varphi(x, y) \leq 0
\end{aligned}\right.
$$

where $\varphi(x, y)=x-y x^{2}$. The switching curve is given by $\Sigma=\varphi^{-1}(0) \cap U=\{(x, y) \in$ $\left.\mathbb{R}^{2} ; y=1 / x\right\}$. As before, we have four vector fields determined by the implicit differential equations:

$$
\begin{aligned}
& V_{1+}(x, y)=\frac{\partial}{\partial x}+\sqrt{x} \frac{\partial}{\partial y}, \\
& V_{1-}(x, y)=\frac{\partial}{\partial x}-\sqrt{x} \frac{\partial}{\partial y}, \\
& V_{2+}(x, y)=\frac{\partial}{\partial x}+\sqrt{y x^{2}} \frac{\partial}{\partial y}, \\
& V_{2-}(x, y)=\frac{\partial}{\partial x}-\sqrt{y x^{2}} \frac{\partial}{\partial y} .
\end{aligned}
$$

The sign of the Lie derivative $V_{i} . \varphi$, with $i \in\{1,2\}$ and $(x, y) \in \Sigma$ determines the regions on $\Sigma$. Note that if $\left(x, h_{t}(x)\right) \in \Sigma$ then $\left(x, h_{t}(x)\right)=\left(x, \frac{1}{x}\right)$. We have $\left(V_{1,+}\right) \cdot \varphi=\left(V_{2,+}\right) \cdot \varphi=$ $-x^{5 / 2}-1$ and $\left(V_{1,-}\right) \cdot \varphi=\left(V_{2,-}\right) \cdot \varphi=x^{5 / 2}-1$.

(a) If $i=+, j=+$ then $\left[V_{1, i} . \varphi\right]\left[V_{2, j} . \varphi\right]>0$ for all $(x, y) \in \Sigma$. Therefore $\Sigma_{s w}=$ $\Sigma, \Sigma_{s l}=\emptyset$ and $\Sigma_{e s}=\emptyset$.

(b) If $i=+, j=-$ then $\left[V_{1, i} . \varphi\right]\left[V_{2, j} . \varphi\right]>0$ for all $(x, y) \in \Sigma_{1}$ and $\left[V_{1, i} . \varphi\right]\left[V_{2, j} . \varphi\right]<0$ and $\left[V_{1, i} . \varphi\right]<0$ for all $(x, y) \in \Sigma_{2}$. Therefore $\Sigma_{s w}=\Sigma_{1}, \Sigma_{s l}=\Sigma_{2}$ and $\Sigma_{e s}=\emptyset$.

(c) If $i=-, j=+$ then $\left[V_{1, i} . \varphi\right]\left[V_{2, j} . \varphi\right]>0$ for all $(x, y) \in \Sigma_{1}$ and $\left[V_{1, i} . \varphi\right]\left[V_{2, j} . \varphi\right]<0$ and $\left[V_{2, j} . \varphi\right]<0$ for all $(x, y) \in \Sigma_{2}$. Therefore $\Sigma_{s w}=\Sigma_{1}, \Sigma_{s l}=\emptyset$ and $\Sigma_{e s}=\Sigma_{2}$.

(d) If $i=-, j=-$ then $\left[V_{1, i} . \varphi\right]\left[V_{2, j} . \varphi\right]>0$ for all $(x, y) \in \Sigma$. Therefore $\Sigma_{s w}=$ $\Sigma, \Sigma_{s l}=\emptyset$ and $\Sigma_{e s}=\emptyset$.

Therefore, we conclude the veracity of the data presented in Table 6 for case $t=C W$.

If $t=F W$ Consider the discontinuous system $S=\left(S_{1}, S_{2}\right)$ :

$$
S=\left\{\begin{aligned}
\left(\dot{x}-1, \dot{y}^{2}-x\right) & =(0,0), \text { if } \varphi(x, y) \geq 0 \\
\left(\dot{x}-1, \dot{y}^{2}-x(x-y)^{2}\right) & =(0,0), \text { if } \varphi(x, y) \leq 0
\end{aligned}\right.
$$

where $\varphi(x, y)=x-x(x-y)^{2}$. The switching curve is given by $\Sigma=\varphi^{-1}(0) \cap U=$ $\left\{(x, y) \in \mathbb{R}^{2} ; y=x+1, y=x-1\right\}$.

As before, we have four vector fields determined by the implicit differential equations:

$$
\begin{aligned}
& V_{1+}(x, y)=\frac{\partial}{\partial x}+\sqrt{x} \frac{\partial}{\partial y}, \\
& V_{1-}(x, y)=\frac{\partial}{\partial x}-\sqrt{x} \frac{\partial}{\partial y}, \\
& V_{2+}(x, y)=\frac{\partial}{\partial x}+\sqrt{x(x-y)^{2}} \frac{\partial}{\partial y}, \\
& V_{2-}(x, y)=\frac{\partial}{\partial x}-\sqrt{x(x-y)^{2}} \frac{\partial}{\partial y} .
\end{aligned}
$$

The signs of the Lie derivative $V_{i} . \varphi$, with $i \in\{1,2\}$ and $(x, y) \in \Sigma$ determines the sliding and sewing regions on $\Sigma=\overline{r_{1}} \cup \overline{r_{2}} \cup \overline{r_{3}} \cup \overline{r_{4}}$. where $r_{1}=\{(x, x+1) \in \Sigma: x<1\}, r_{2}=$ 
$\{(x, x+1) \in \Sigma: x>1\}, r_{3}=\{(x, x-1) \in \Sigma: x<1\}$ and $r_{4}=\{(x, x-1) \in \Sigma: x>1\}$. Note that if $(x, y) \in r_{1} \cup r_{2}$ then $(x, y)=(x, x+1)$. We have $\left(V_{1,+}\right) \cdot \varphi=\left(V_{2,+}\right) \cdot \varphi=$ $-2 x(\sqrt{x}-1)$ and $\left(V_{1,-}\right) \cdot \varphi=\left(V_{2,-}\right) \cdot \varphi=2\left(x^{3 / 2}+x\right)$.

(a) If $i=+, j=+$ then $\left[V_{1, i} . \varphi\right]\left[V_{2, j} . \varphi\right]>0$ for all $(x, y) \in r_{1} \cup r_{2}$. Therefore $\Sigma_{s w}=$ $r_{1} \cup r_{2}, \Sigma_{s l}=\emptyset$ and $\Sigma_{e s}=\emptyset$.

(b) If $i=+, j=-$ then $\left[V_{1, i} . \varphi\right]\left[V_{2, j} . \varphi\right]>0$ for all $(x, y) \in r_{1}$ and $\left[V_{1, i} . \varphi\right]\left[V_{2, j} . \varphi\right]<0$ and $\left[V_{1, i} . \varphi\right]<0$ for all $(x, y) \in r_{2}$ Therefore $\Sigma_{s w}=r_{1}, \Sigma_{s l}=r_{2}$ and $\Sigma_{e s}=\emptyset$.

(c) If $i=-, j=+$ then $\left[V_{1, i} . \varphi\right]\left[V_{2, j} . \varphi\right]>0$ for all $(x, y) \in r_{1}$ and $\left[V_{1, i} . \varphi\right]\left[V_{2, j} . \varphi\right]<0$ and $\left[V_{2, j} . \varphi\right]<0$ for all $(x, y) \in r_{2}$. Therefore $\Sigma_{s w}=r_{1}, \Sigma_{s l}=\emptyset$ and $\Sigma_{e s}=r_{2}$.

(d) If $i=-, j=-$ then $\left[V_{1, i} . \varphi\right]\left[V_{2, j} . \varphi\right]>0$ for all $(x, y) \in r_{1} \cup r_{2}$. Therefore $\Sigma_{s w}=$ $r_{1} \cup r_{2}, \Sigma_{s l}=\emptyset$ and $\Sigma_{e s}=\emptyset$.

Analogously if $(x, y) \in r_{3} \cup r_{4}$ then $(x, y)=(x, x-1)$. We have $\left(V_{1,+}\right) \cdot \varphi=\left(V_{2,+}\right) \cdot \varphi=$ $2 x(\sqrt{x}-1)$ and $\left(V_{1,-}\right) \cdot \varphi=\left(V_{2,-}\right) \cdot \varphi=-2\left(x^{3 / 2}+x\right)$.

(a) If $i=+, j=+$ then $\left[V_{1, i} . \varphi\right]\left[V_{2, j} . \varphi\right]>0$ for all $(x, y) \in r_{3} \cup r_{4}$. Therefore $\Sigma_{s w}=$ $r_{3} \cup r_{4}, \Sigma_{s l}=\emptyset$ and $\Sigma_{e s}=\emptyset$.

(b) If $i=+, j=-$ then $\left[V_{1, i} . \varphi\right]\left[V_{2, j} . \varphi\right]>0$ for all $(x, y) \in r_{3}$ and $\left[V_{1, i} . \varphi\right]\left[V_{2, j} . \varphi\right]<0$ and $\left[V_{1, i} . \varphi\right]<0$ for all $(x, y) \in r_{4}$. Therefore $\Sigma_{s w}=r_{3}, \Sigma_{s l}=r_{4}$ and $\Sigma_{e s}=\emptyset$.

(c) If $i=-, j=+$ then $\left[V_{1, i} \cdot \varphi\right]\left[V_{2, j} \cdot \varphi\right]>0$ for all $(x, y) \in r_{3}$ and $\left[V_{1, i} \cdot \varphi\right]\left[V_{2, j} \cdot \varphi\right]<0$ and $\left[V_{2, j} . \varphi\right]<0$ for all $(x, y) \in r_{4}$. Therefore $\Sigma_{s w}=r_{3}, \Sigma_{s l}=\emptyset$ and $\Sigma_{e s}=r_{4}$.

(d) If $i=-, j=-$ then $\left[V_{1, i} . \varphi\right]\left[V_{2, j} . \varphi\right]>0$ for all $(x, y) \in r_{3} \cup r_{4}$. Therefore $\Sigma_{s w}=$ $r_{3} \cup r_{4}, \Sigma_{s l}=\emptyset$ and $\Sigma_{e s}=\emptyset$.

Denoting $\Sigma_{1}=r_{1} \cup r_{3}, \Sigma_{2}=r_{2} \cup r_{4}$ and $\Sigma=\bar{\Sigma}_{1} \cup \bar{\Sigma}_{2}$ we conclude the following.

(a) If $i=+, j=+$ then $\left[V_{1, i} \cdot \varphi\right]\left[V_{2, j} \cdot \varphi\right]>0$ for all $(x, y) \in \Sigma$. Therefore $\Sigma_{s w}=$ $\Sigma, \Sigma_{s l}=\emptyset$ and $\Sigma_{e s}=\emptyset$.

(b) If $i=+, j=-$ then $\left[V_{1, i} . \varphi\right]\left[V_{2, j} . \varphi\right]>0$ for all $(x, y) \in \Sigma_{1}$ and $\left[V_{1, i} . \varphi\right]\left[V_{2, j} . \varphi\right]<0$ and $\left[V_{1, i} . \varphi\right]<0$ for all $(x, y) \in \Sigma_{2}$. Therefore $\Sigma_{s w}=\Sigma_{1}, \Sigma_{s l}=\Sigma_{2}$ and $\Sigma_{e s}=\emptyset$.

(c) If $i=-, j=+$ then $\left[V_{1, i} . \varphi\right]\left[V_{2, j} . \varphi\right]>0$ for all $(x, y) \in \Sigma_{1}$ and $\left[V_{1, i} . \varphi\right]\left[V_{2, j} . \varphi\right]<0$ and $\left[V_{2, j} . \varphi\right]<0$ for all $(x, y) \in \Sigma_{2}$. Therefore $\Sigma_{s w}=\Sigma_{1}, \Sigma_{s l}=\emptyset$ and $\Sigma_{e s}=\Sigma_{2}$.

(d) If $i=-, j=-$ then $\left[V_{1, i} \cdot \varphi\right]\left[V_{2, j} \cdot \varphi\right]>0$ for all $(x, y) \in \Sigma$. Therefore $\Sigma_{s w}=$ $\Sigma, \Sigma_{s l}=\emptyset$ and $\Sigma_{e s}=\emptyset$.

Thus the veracity of the data presented in Table 6 is proved for case $t=F W$.

Proof of Theorem 5 Let $\left(S_{1}, S_{2}\right)$ be a discontinuous system with $\frac{\partial \varphi}{\partial y}(x, y) \neq 0$, for any $(x, y) \in \Sigma_{s}=\Sigma_{s l} \cup \Sigma_{e s}$. Consider the vectors in the cone generated by $V_{1, i}$ and $V_{2, j}$ with $i \neq j, i, j \in\{+,-\}$ defined as follows

$$
\left(1, i \sqrt{g_{1}}+\lambda\left(j \sqrt{g_{2}}-i \sqrt{g_{1}}\right)\right)
$$

If

$$
\left\langle\left(1, i \sqrt{g_{1}}+\lambda\left(j \sqrt{g_{2}}-i \sqrt{g_{1}}\right)\right) \cdot\left(\frac{\partial \varphi}{\partial x}, \frac{\partial \varphi}{\partial y}\right)\right\rangle=0
$$

then

$$
\frac{\partial \varphi}{\partial x}+i \sqrt{g_{1}} \frac{\partial \varphi}{\partial y}+\lambda\left(j \sqrt{g_{2}}-i \sqrt{g_{1}}\right) \frac{\partial \varphi}{\partial y}=0
$$


and so

$$
\lambda=\frac{-\frac{\partial \varphi}{\partial x}-i \sqrt{g_{1}} \frac{\partial \varphi}{\partial y}}{\left(j \sqrt{g_{2}}-i \sqrt{g_{1}}\right) \frac{\partial \varphi}{\partial y}} .
$$

Replacing in (12), we have the sliding vector fields

$$
\left(V_{1+}, V_{2-}\right)_{s}=\left(V_{1-}, V_{2+}\right)_{s}=\left(1,-\frac{\partial \varphi}{\partial x}(x, y) / \frac{\partial \varphi}{\partial y}(x, y)\right)
$$

\section{Discontinuous Implicit Differential Systems and Singular Perturbation}

In this section we proceed the $\psi$-regularization of discontinuous implicit differential systems and get an implicit singular perturbation problem (we refer [10] for an introduction to the general theory of singular perturbation).

Let $U \subset \mathbb{R}^{2}$ be an open subset and $\varepsilon \geq 0$. An implicit singular perturbation problem in $U$ (ISP-problem) is a differential system which can be written like

$$
\dot{x}=f(x, y, \varepsilon), \quad(\varepsilon \dot{y})^{2}=h(x, y, \varepsilon)
$$

or equivalently, after the time rescaling $\tau=t / \varepsilon$

$$
\frac{d x}{d \tau}=\varepsilon f(x, y, \varepsilon), \quad\left(\frac{d y}{d \tau}\right)^{2}=h(x, y, \varepsilon)
$$

with $(x, y) \in U$ and $f, h$ are smooth in all variables.

Note that two different timescales can be derived: the slow timescale $t$ and the fast timescale $\tau=t / \varepsilon$. Systems (13),(14) are called slow and fast systems, respectively. Taking $\varepsilon=0$ in (13) and in (14) we obtain two systems with dynamics essentially different: the reduced problem

$$
\dot{x}=f(x, y, 0), \quad 0=h(x, y, 0)
$$

and the layer problem

$$
\frac{d x}{d \tau}=0, \quad\left(\frac{d y}{d \tau}\right)^{2}=h(x, y, 0) .
$$

Consider $\mathcal{V}=\{(x, y): h(x, y, 0)=0\}$. We call $\mathcal{V}$ the slow manifold of the implicit singular perturbation problem. Observe that (15) defines a dynamical system on $\mathcal{V}$. On the other hand, $\mathcal{V}$ is a manifold of singular points for (16). Combining results on the dynamics of these two limiting problems, with $\varepsilon=0$, one obtains information on the dynamics of (13) for small values of $\varepsilon$.

Now we introduce a tool in the study of non-smooth implicit differential systems. Essentially we adapt the regularization process of non-smooth vector fields, introduced by Sotomayor and Teixeira, for our case. As before, a $\mathbb{C}^{\infty}$ function $\psi: \mathbb{R} \rightarrow \mathbb{R}$ is a transition function if $\psi(t)=-1$ for $t \leq-1, \psi(t)=1$ for $t \geq 1$ and $\psi^{\prime}(t)>0$ if $t \in(-1,1)$. The $\psi$-regularization of $F=\left(S_{1}, S_{2}\right) \in \Omega, U \subset \mathbb{R}^{m}$, where

$$
S_{i}(x, y, \dot{x}, \dot{y})=\left(\dot{x}-1, \dot{y}^{2}-g_{i}(x, y)\right)=(0,0), \quad i=1,2 ;
$$


is the one-parameter family $S_{\varepsilon} \in C^{n}$ given by

$$
S_{\varepsilon}(q, \dot{q})=\left(\dot{x}-1, \dot{y}^{2}-\left(\frac{g_{1}+g_{2}}{2}+\psi_{\varepsilon}(\varphi(q)) \frac{g_{1}-g_{2}}{2}\right)\right)
$$

with $\psi_{\varepsilon}(t)=\psi\left(\frac{t}{\varepsilon}\right)$, for $\varepsilon>0$.

Next theorem says that the $\psi$-regularization of discontinuous implicit differential systems provides an ISP. In general this transition can be done explicitly.

Theorem 6 Consider $S=\left(S_{1}, S_{2}\right) \in \Omega, S_{\varepsilon}$ its $\psi$-regularization and $q \in \Sigma$. Suppose that $\psi$ is a polynomial of degree $2 \ell$ in a small interval $0 \in I \subset(-1,1)$. Then the trajectories of $S_{\varepsilon}$ in $U_{\varepsilon}=\{q \in U ; \varphi(q) / \varepsilon \in I\}$ are solutions of a ISP-problem.

Proof Let $S \in \Omega$ with $S_{i}(x, y, \dot{x}, \dot{y})=\left(\dot{x}-1, \dot{y}^{2}-g_{i}(x, y)\right)=(0,0), \quad i=1,2$. Suppose that $a_{1} t+\ldots+a_{2 \ell} t^{2 \ell}$ is the polynomial expression of $\psi$ on $I \subset \mathbb{R}$ with $0 \in I$. The trajectories of $S_{\varepsilon}$ on $U_{\varepsilon}$ are the solutions the of implicit differential system

$$
\dot{x}=1 ; \quad \dot{y}^{2}=\left(\frac{g_{1}+g_{2}}{2}+\psi_{\epsilon}(\varphi) \frac{g_{1}-g_{2}}{2}\right) .
$$

The time rescaling $\tau=t / \epsilon^{\ell}$ gives

$$
\begin{aligned}
x^{\prime} & =\varepsilon^{\ell} ; \\
\left(y^{\prime}\right)^{2} & =\varepsilon^{2 \ell}\left(\frac{g_{1}+g_{2}}{2}\right)+\varepsilon^{2 \ell}\left(\frac{a_{1} \varphi}{\varepsilon}+\ldots+\frac{a_{2 \ell} \varphi^{2 \ell}}{\varepsilon^{2 \ell}}\right)\left(\frac{g_{1}-g_{2}}{2}\right) .
\end{aligned}
$$

Remark 1 For each surface $p^{2}=g_{i}(x, y), i=1,2$ we have defined a vector field $\xi_{i}$, according (6). Besides, for the surface $p^{2}-\left(\frac{g_{1}+g_{2}}{2}+\psi_{\varepsilon}(\varphi(q)) \frac{g_{1}-g_{2}}{2}\right)=0$ we can also consider the vector field $\xi_{\varepsilon}$. It is easy to see that

$$
\Pi^{-1} R\left(\Pi\left(\xi_{1}\right), \Pi\left(\xi_{2}\right)\right)=\xi_{\varepsilon}
$$

where $\Pi(x, y, p)=(x, p)$, and $R(X)$ is the $\psi$-regularization of $X=\left(X_{1}, X_{2}\right) \in \Lambda$ defined in (10).

Acknowledgements The authors are partially supported by CAPES, CNPq-Brazil, FAPESP and FP7PEOPLE-2012-IRSES 318999.

\section{References}

1. Arnold, V.I.: Geometrical methods in the theory of ordinary differential equations, 2nd edn. Springer, New york (1987)

2. Banerjee, S., Verghese, G.C.: Nonlinear Phenomena in Power Electronics: Attractors, Bifurcations, Chaos, and Nonlinear Control. IEEE Press, Piscataway (2001)

3. Begely, C.J., Virgin, L.N.: Grazing bifurcations and basins of attraction in an impact-friction oscillator. Physica D 130, 43-57 (1999)

4. Brogliato, B.B., Heemels, W.P.M.H.: The complementarity class of hybrid dynamical systems. Eur. J. Control. 9, 311-319 (2003)

5. Bruce, J.W., Tari, F.: Duality and implicit differential equations. Nonlinearity 13, 791-811 (2000)

6. Buzzi, C., Silva, P.R., Teixeira, M.A.: A Singular approach to discontinuous vector fields on the plane. J. Differ. Equ. 231, 633-655 (2006)

7. Davydov, A.A.: Qualitative theory of control systems. Translation of mathematical monographs, vol. 141. American Mathematical Society, Providence (1994) 
8. Davydov, A.A., Ishikawa, G., Izumiya, S., Sun, W.Z.: Generic singularities of implicit systems of first order differential equations on the plane. Jpn. J. Math. 3, 93-119 (2008)

9. di Bernardo, M., Budd, C.J., Champneys, A.R., Kowalczyk, P.: Piecewise-smooth dynamical systems. Theory and aplications. Applied Mathematical Science, vol. 163. Springer, London (2008)

10. Fenichel, N.: Geometric singular perturbation theory for ordinary differential equations. J. Differ. Equ. 31, 53-98 (1979)

11. Filippov, A.F.: Differential equations with discontinuous right-hand sides, Mathematics and its Applications (Soviet Series). Kluwer Academic Publishers, Dordrecht (1988)

12. Fossas, E., Hogan, S.J., Seara, T.M.: Two-parameter bifurcation curves in power electronic converters. Int. J. Bifur. Chaos Appl. Sci. Eng. 19, 349-357 (2009)

13. Llibre, J., Silva, P.R., Teixeira, M.A.: Regularization of discontinuous vector fields via singular perturbation. J. Dyn. Differ. Equ. 19(2), 309-331 (2007)

14. Llibre, J., Silva, P.R., Teixeira, M.A.: Sliding vector fields via slow fast systems. Bull. Belg. Math. Soc. Simon Stevin 15, 851-869 (2008)

15. Llibre, J., Silva, P.R., Teixeira, M.A.: Study of singularities in non smooth dynamical systems via singular perturbation. SIAM J. Appl. Dyn. Syst. 8, 508-526 (2009)

16. Llibre, J., Teixeira, M.A.: Regularization of discontinuous vector fields in dimension three. Discret. Contin. Dyn. Syst. 3, 235-241 (1997)

17. Sotomayor, J., Teixeira, M.A.: Regularization of discontinuous vector fields. In: International conference on differential equations, vol. 95, pp. 207-223. Equadiff, Lisboa (1996)

18. Takens, F.: Implicit differential equations; some open problems. Singularités d'applications différentiables (Sém., Plans-sur-Bex. Lecture Notes in Math, vol. 535, pp. 237-253. Springer, Berlin (1976) 